Luca Chiantini · Ciro Ciliberto

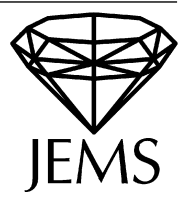

\title{
On the dimension of secant varieties
}

Received January 31, 2008 and in revised form October 8, 2008

\begin{abstract}
In this paper we generalize Zak's theorems on tangencies and on linear normality as well as Zak's definition and classification of Severi varieties. In particular we find sharp lower bounds for the dimension of higher secant varieties of a given variety $X$ under suitable regularity assumptions on $X$, and we classify varieties for which the bound is attained.
\end{abstract}

Keywords. Higher secant varieties, tangential projections, special varieties

\section{Contents}

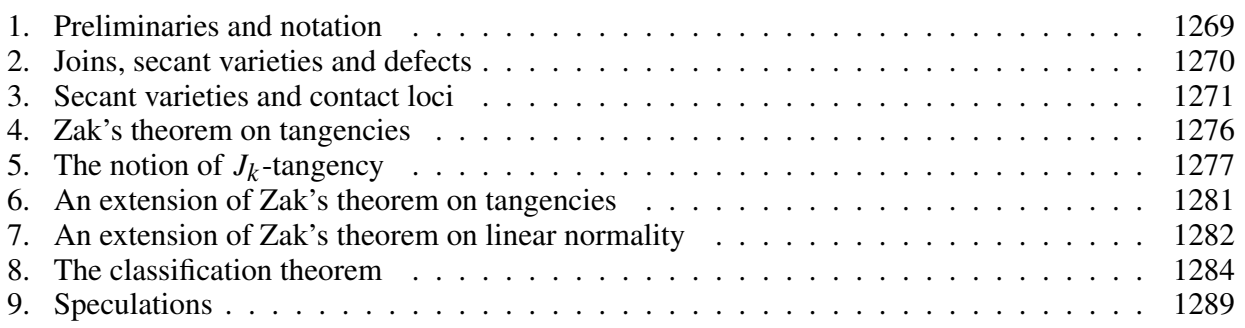

\section{Introduction}

Let $X \subset \mathbb{P}^{r}$ be an irreducible, projective, non-degenerate variety of dimension $n$. For any non-negative integer $k$ one can consider the $k$-secant variety of $X$, which is the Zariski closure in $\mathbb{P}^{r}$ of the union of all $k$-dimensional subspaces of $\mathbb{P}^{r}$ that are spanned by $k+$ 1 independent points of $X$. Secant varieties are basic projective invariants related to a given variety $X$ and their understanding is of primary importance in the study of the geometry and topology of $X$. As such, they have been, for more than a century, the object

L. Chiantini: Dipartimento di Scienze Matematiche e Informatiche, Pian Dei Mantellini 44, I-53100 Siena, Italy; e-mail: chiantini@unisi.it, url: http://www.mat.unisi.it/newsito/docente.php?id=4

C. Ciliberto: Dipartimento di Matematica, Università di Roma Tor Vergata,

Via della Ricerca Scientifica, 00133 Roma, Italy; e-mail: cilibert@axp.mat.uniroma2.it, url: http://www.mat.uniroma2.it//cilibert/

Mathematics Subject Classification (2010): Primary 14N05; Secondary 14C20 
of important research in algebraic geometry. For instance, the classification of defective varieties, i.e. the ones for which some secant variety has dimension smaller than expected, goes back to several classical authors, like Terracini [25], Palatini [18], [19], and Scorza [22], [23] to mention but a few. For recent developments on this classical theme see [5], [6], [7] and the basic reference [27].

In more recent times the interest in the geometry of projective varieties has been revived by Zak's epochal work (see [27]). Specifically, Zak first proved his so-called theorem on tangencies, a basic tool which, although very classical in spirit, completely escaped the consideration of the classics. This theorem was used by Zak to prove a sharp lower bound for the dimension of the first secant variety to a smooth variety $X$, as well as the classification of those varieties achieving the bound, i.e. the so-called Severi varieties.

In this paper we present an extension of these results of Zak's. Namely we first extend the theorem on tangencies, then we provide, under suitable regularity assumptions on a variety $X$, a lower bound for the dimension of its higher secant varieties, and finally we classify the varieties for which the bound is attained.

To be specific, we introduce in $\$ 5$ the notion of $J_{k}$-tangency extending the concept of $J$-tangency which is one of the cornerstones of Zak's theorem on tangencies. The notion of $J_{k}$-tangency is crucial for us, so we devote to it, and to related concepts, all $\$ 5$ In $\$ 6$ we prove Theorem 6.1, which is the announced generalization of the theorem on tangencies. In $\$ 7$ we prove our extension of Zak's theorem on linear normality, i.e. Theorem 7.5 providing a sharp lower bound for the dimension of the $k$-secant variety to varieties having a suitable tangential behaviour which we call $R_{k}$-property, where $R$ stands for regularity (see Definition 5.5). Basic tools in the proof are the generalized theorem on tangencies, as well as a few basic facts about secant varieties, defects and contact loci, which we present in $\$ \$ 2$ and 3 .

Notice that, without suitable regularity assumptions, it seems quite unlikely to get good bounds for the dimension of higher secant varieties. Examples, together with a nice account of the general theory, can be found in [17], where several partial results are given.

In $\$ 8$ we define $k$-Severi varieties as the irreducible $R_{k}$-varieties for which the bound in the extended theorem on linear normality is attained. Smoothness is not required in the definition. However we prove that $k$-Severi varieties are smooth (see Theorem 8.7). The classification of $k$-Severi varieties is given in Theorem 8.3 The main point here is to observe that $k$-Severi varieties are Scorza varieties in the sense of Zak (see [27, Chapter VI]). Then our classification theorem follows from Zak's classification of Scorza varieties in [27]. However, a crucial point here is the smoothness of certain contact loci (ensured by Lemma 8.5 and Claims 8.9 and 8.12 in the proof of Theorem 8.7), which is essential in Zak's analysis of Scorza varieties. It is well known that strong motivations for Zak's work have been Hartshorne's conjectures. One of them, Hartshorne's Conjecture 7.2 on linear normality, has been proved by Zak. The other (see Conjecture 9.1) is still unsolved. In $\$ 9$ we speculate on a possible extension of this conjecture which may be suggested by the results of the present paper.

We want to finish by observing that, besides the intrinsic interest of the subject, defective varieties, or more generally properties of secant varieties, are relevant also in other fields of mathematics, such as expressions of polynomials as sums of powers, Waring 
type problems, polynomial interpolation, rank tensor computations and canonical forms, Bayesian networks, algebraic statistics etc. (see [9] as a general reference, [4], [13], [15], [20]). This classical subject is therefore still very lively and widely open to future research.

\section{Preliminaries and notation}

We work over the field $\mathbb{C}$ of complex numbers and we consider the projective space $\mathbb{P}^{r}=\mathbb{P}_{\mathbb{C}}^{r}$, equipped with the tautological line bundle $\mathscr{O}_{\mathbb{P} r}(1)$.

If $Y \subset \mathbb{P}^{r}$ is a subset, we denote by $\langle Y\rangle$ the span of $Y$. We say that $Y$ is non-degenerate if $\langle Y\rangle=\mathbb{P}^{r}$. A linear subspace of dimension $n$ of $\mathbb{P}^{r}$ will be called an $n$-subspace of $\mathbb{P}^{r}$.

Given a subscheme $X \subset \mathbb{P}^{r}, I_{X}$ will denote its homogeneous ideal and $\mathscr{I}_{X}$ the ideal sheaf of $X$.

Let $X \subseteq \mathbb{P}^{r}$ be a scheme. By a general point of $X$ we mean a point varying in some dense open Zariski subset of some irreducible component of $X$. We will denote by $\operatorname{dim}(X)$ the maximum of the dimensions of the irreducible components of $X$. We will often assume that $X$ is pure, i.e. all the irreducible components of $X$ have the same dimension. If $X$ is projective, reduced and pure, we will say it is a variety.

Let $X \subset \mathbb{P}^{r}$ be a variety. We will denote by $\operatorname{Sing}(X)$ the closed Zariski subset of singular points of $X$. Let $x \in X-\operatorname{Sing}(X)$ be a smooth point. We will denote by $T_{X, x}$ the embedded tangent space to $X$ at $x$, which is an $n$-subspace of $\mathbb{P}^{r}(n=\operatorname{dim}(X))$. More generally, if $x_{1}, \ldots, x_{k}$ are smooth points of $X$, we will set

$$
T_{X, x_{1}, \ldots, x_{k}}=\left\langle\bigcup_{i=1}^{k} T_{X, x_{i}}\right\rangle .
$$

We will denote by $V_{n, d}$ the $d$-Veronese variety of $\mathbb{P}^{n}$, i.e. the image of $\mathbb{P}^{n}$ via the $d$-Veronese embedding

$$
v_{n, d}: \mathbb{P}^{n} \rightarrow \mathbb{P}^{r}, \quad r=\left(\begin{array}{c}
n+d \\
d
\end{array}\right)-1
$$

Given positive integers $0<m_{1} \leq \cdots \leq m_{h}$ we will denote by $\operatorname{Seg}\left(m_{1}, \ldots, m_{h}\right)$ the Segre variety of type $\left(m_{1}, \ldots, m_{h}\right)$, i.e. the image of $\mathbb{P}^{m_{1}} \times \cdots \times \mathbb{P}^{m_{h}}$ in $\mathbb{P}^{r}, r=$ $\left(m_{1}+1\right) \ldots\left(m_{h}+1\right)-1$, via the Segre embedding

$$
s_{m_{1}, \ldots, m_{h}}: \mathbb{P}^{m_{1}} \times \cdots \times \mathbb{P}^{m_{h}} \rightarrow \mathbb{P}^{r} .
$$

Let $0 \leq a_{1} \leq \cdots \leq a_{n}$ be integers and set $\mathbb{P}\left(a_{1}, \ldots, a_{n}\right):=\mathbb{P}\left(\mathcal{O}_{\mathbb{P}^{1}}\left(a_{1}\right) \oplus \cdots \oplus\right.$ $\left.\mathcal{O}_{\mathbb{P}^{1}}\left(a_{n}\right)\right)$. Set $r=a_{1}+\cdots+a_{n}+n-1$ and consider the morphism

$$
\phi_{a_{1}, \ldots, a_{n}}: \mathbb{P}\left(a_{1}, \ldots, a_{n}\right) \rightarrow \mathbb{P}^{r}
$$

defined by the sections of the line bundle $\mathcal{O}_{\mathbb{P}\left(a_{1}, \ldots, a_{n}\right)}(1)$. We denote the image of $\phi_{a_{1}, \ldots, a_{n}}$ by $S\left(a_{1}, \ldots, a_{n}\right)$. As soon as $a_{n}>0$, the morphism $\phi_{a_{1}, \ldots, a_{n}}$ is birational to its image. 
Then the dimension of $S\left(a_{1}, \ldots, a_{n}\right)$ is $n$, its degree is $a_{1}+\cdots+a_{n}=r-n+1$ and $S\left(a_{1}, \ldots, a_{n}\right)$ is a rational normal scroll, which is smooth if and only if $a_{1}>0$.

We will denote by $\mathbb{G}(m, n)$ the Grassmann variety of $m$-subspaces in $\mathbb{P}^{n}$, embedded in $\mathbb{P}^{r}, r=\left(\begin{array}{c}n+1 \\ m+1\end{array}\right)-1$, via the Plücker embedding.

For all integers $k \geq 1$, we will denote by $S_{k}$ the $k$-spinor variety, which parametrizes the family of $(k-1)$-subspaces contained in a smooth quadric of dimension $2 k-1$. The variety $S_{k}$ is smooth, of dimension $\left(\begin{array}{c}k+1 \\ 2\end{array}\right)$, and its Picard group is generated by a very ample divisor which embeds $S_{k}$ in $\mathbb{P}^{2^{k}-1}$.

\section{Joins, secant varieties and defects}

Let $X_{0}, \ldots, X_{k}$ be varieties in $\mathbb{P}^{r}$. The join $J\left(X_{0}, \ldots, X_{k}\right)$ of $X_{0}, \ldots, X_{k}$ is the closure in $\mathbb{P}^{r}$ of the set

$\left\{x \in \mathbb{P}^{r}: x\right.$ lies in the span of $k+1$ independent points $\left.p_{i} \in X_{i}, 0 \leq i \leq k\right\}$.

We will use the exponential notation $J\left(X_{1}^{m_{1}}, \ldots, X_{h}^{m_{h}}\right)$ if $X_{i}$ is repeated $m_{i}$ times, $1 \leq$ $i \leq h$. If $X_{0}, \ldots, X_{k}$ are irreducible, their join is also irreducible. The definition is independent of the order of $X_{0}, \ldots, X_{k}$ and one has

$$
\operatorname{dim}\left(J\left(X_{0}, \ldots, X_{k}\right)\right) \leq \min \left\{r, k+\sum_{i=0}^{k} \operatorname{dim}\left(X_{i}\right)\right\} .
$$

The right hand side is called the expected dimension of the join.

If $X$ is irreducible of dimension $n$, we will set $S^{k}(X)=J\left(X^{k+1}\right)$, and we will call $S^{k}(X)$ the $k$-secant variety of $X$. This is an irreducible variety of dimension

$$
s^{(k)}(X):=\operatorname{dim}\left(S^{k}(X)\right) \leq \min \{r, n(k+1)+k\}=: e^{(k)}(X) .
$$

Again, the right hand side is called the expected dimension of $S^{k}(X)$.

If $X$ is reducible of dimension $n$, then $J\left(X^{k+1}\right)$ is in general reducible and not pure. In this case, we consider the union of all joins $J\left(X_{0}, \ldots, X_{k}\right)$, where $X_{0}, \ldots, X_{k}$ are distinct irreducible components of $X$. It is convenient for us to denote this by $S^{k}(X)$ and call it the $k$-secant variety of $X$. With this convention, formula (2.1) still holds. The varieties $X$ we will be considering next, even if reducible, will have the property that $S^{k}(X)$ is pure. We will therefore often make this assumption.

One says that $X$ is $k$-defective when strict inequality holds in 2.1). One calls

$$
\delta_{k}(X):=e^{(k)}(X)-s^{(k)}(X)
$$

the $k$-secant defect of $X$. There is however a slightly different concept of $k$-defect, which will be useful for us, i.e. the concept of $k$-fibre defect $f_{k}(X)$, defined as

$$
f_{k}(X)=(k+1) n+k-s^{(k)}(X) .
$$

Notice that $f_{k}(X)=\delta_{k}(X)$ if $r \geq(k+1) n+k$, while otherwise $f_{k}(X)=\delta_{k}(X)+$ $(k+1) n+k-r$, thus $f_{k}(X)$ can be positive even if $\delta_{k}(X)=0$. 
Remark 2.1. The reason for the name fibre defect is the following. Assume $S^{k}(X)$ is pure. Then $f_{k}(X)$ equals the dimension of the family of $(k+1)$-secant $k$-spaces to $X$ passing through a general point of $S^{k}(X)$.

Indeed, consider the abstract secant variety $\mathcal{S}^{k}(X)$ which is the union of the closures of the sets

$$
\left\{\left(p_{0}, \ldots, p_{k}, x\right) \in X_{0} \times \cdots \times X_{k} \times \mathbb{P}^{r}: x \in\left\langle p_{0}, \ldots, p_{k}\right\rangle \simeq \mathbb{P}^{k}\right\}
$$

with $X_{0}, \ldots, X_{k}$ distinct irreducible components of $X$. Then the image of the projection $p: \mathcal{S}^{k}(X) \rightarrow \mathbb{P}^{r}$ is $S^{k}(X)$ and $f_{k}(X)$ is the dimension of a general fibre of $p$. Hence one may have $f_{k}(X)>0$ even if $X$ is not $k$-defective: this happens when $S^{k}(X)=\mathbb{P}^{r}$ and $r<(k+1) n+k$.

We will use abbreviated notation like $s^{(k)}, e^{(k)}, \delta_{k}, f_{k}$ instead of $s^{(k)}(X), e^{(k)}(X)$, $\delta_{k}(X), f_{k}(X)$ if there is no danger of confusion. Also, we may drop the index $k$ when $k=1$.

\section{Secant varieties and contact loci}

If $X_{0}, \ldots, X_{k}$ are projective varieties in $\mathbb{P}^{r}$, then Terracini's Lemma describes the tangent space to their join at a general point of it (see [25] or, for modern versions, [1], [5], [8], [10], [27]).

Theorem 3.1. Let $X_{0}, \ldots, X_{k}$ be varieties in $\mathbb{P}^{r}$. If $p_{i} \in X_{i}, 0 \leq i \leq k$, are general points and $x \in\left\langle p_{0}, \ldots, p_{k}\right\rangle$ is a general point, then

$$
T_{J\left(X_{0}, \ldots, X_{k}\right), x}=\left\langle T_{X_{0}, p_{0}}, \ldots, T_{X_{k}, p_{k}}\right\rangle .
$$

In particular, if $X \subset \mathbb{P}^{r}$ is an irreducible, projective variety, and if $p_{0}, \ldots, p_{k} \in X$ are general points and $x \in\left\langle p_{0}, \ldots, p_{k}\right\rangle$ is a general point, then

$$
T_{S^{k}(X), x}=T_{X, p_{0}, \ldots, p_{k}} .
$$

We recall a useful consequence of Terracini's Lemma, which is well known in the irreducible case (see [27, p. 106]). The easy proof can be left to the reader.

Proposition 3.2. Let $X \subset \mathbb{P}^{r}$ be a non-degenerate variety. If we have $\operatorname{dim}\left(J\left(X^{k}\right)\right)=$ $\operatorname{dim}\left(J\left(X^{k+1}\right)\right)$ then $J\left(X^{k}\right)=\mathbb{P}^{r}$. Similarly, if $\operatorname{dim}\left(S^{k}(X)\right)=\operatorname{dim}\left(S^{k-1}(X)\right)$, then $S^{k}(X)=\mathbb{P}^{r}$.

Given a variety $X \subset \mathbb{P}^{r}$ of dimension $n$, the Gauss map of $X$ is the rational map

$$
g_{X}: X \rightarrow \mathbb{G}(n, r)
$$

defined at the smooth points of $X$ by mapping $x \in X-\operatorname{Sing}(X)$ to $T_{X, x}$. It is well known that if $x \in X$ is a general point, then the closure of the fibre of $g_{X}$ through $x$ is a linear subspace $\Gamma_{X, x}$ of $\mathbb{P}^{r}$. 
Definition 3.3. In the above setting, $\Gamma_{X, x}$ is called the general Gauss fibre of $X$ and its dimension the tangential defect of $X$, denoted by $t(X)$. We will set $t_{k}(X)=t\left(S^{k}(X)\right)$.

Note that if $X$ is smooth, then $t(X)=0$ (see [27]).

Let $X \subset \mathbb{P}^{r}$ be a non-degenerate variety such that $s^{(k)}(X)<r$. Terracini's Lemma implies that $t_{k}(X) \geq k$. More precise information about $t_{k}(X)$ will be provided in a while. First we are going to introduce a few remarkable families of subvarieties of $X$ related to $S^{k}(X)$.

Given a general point $x \in S^{k}(X)$, i.e. $x \in\left\langle p_{0}, \ldots, p_{k}\right\rangle$, with $p_{0}, \ldots, p_{k} \in X$ general points, consider the Zariski closure of the set

$$
\left\{p \in X-\operatorname{Sing}(X): T_{X, p} \subseteq T_{S^{k}(X), x}\right\} .
$$

We denote by $\Gamma_{p_{0}, \ldots, p_{k}}$ the union of all irreducible components of this locus containing $p_{0}, \ldots, p_{k}$, and by $\gamma_{k}(X)$ its dimension, which clearly does not depend on $p_{0}, \ldots, p_{k}$. Note that $\Gamma_{p_{0}, \ldots, p_{i}} \subseteq \Gamma_{p_{0}, \ldots, p_{k}}$ for all $i=1, \ldots, k$. We set

$$
\Pi_{p_{0}, \ldots, p_{k}}=\left\langle\Gamma_{p_{0}, \ldots, p_{k}}\right\rangle .
$$

We will use the abbreviated notation $\Gamma_{k}, \Pi_{k}, \gamma_{k}$ if no confusion arises. Note that $\Pi_{k}$ contains $\left\langle p_{0}, \ldots, p_{k}\right\rangle$, hence it contains $x$.

Definition 3.4. In the above setting, we will call $\Gamma_{p_{0}, \ldots, p_{k}}$ the tangential $k$-contact locus of $X$ at $p_{0}, \ldots, p_{k}$. We will call $\gamma_{k}(X)$ the $k$-tangential defect of $X$.

Let $X \subset \mathbb{P}^{r}$ be an irreducible, projective variety as above and let again $p_{0}, \ldots, p_{k} \in X$ be general points. Consider the projection of $X$ with centre $T_{X, p_{1}, \ldots, p_{k}}$. We call this a general $k$-tangential projection of $X$, and we denote it by $\tau_{X, p_{1}, \ldots, p_{k}}$, or $\tau_{p_{1}, \ldots, p_{k}}$, or $\tau_{k}$. We denote its image by $X_{p_{1}, \ldots, p_{k}}$, or simply $X_{k}$. By Terracini's Lemma, the map $\tau_{k}$ is generically finite to its image if and only if $s^{(k)}(X)=s^{(k-1)}(X)+n+1$.

Definition 3.5. Let $p_{0} \in X$ be a general point. Let $\Psi_{p_{0}, \ldots, p_{k}}$ be the component of the fibre of $\tau_{X, p_{1}, \ldots, p_{k}}$ containing $p_{0}$. We will denote it by $\Psi_{k}$ if no confusion arises. It is called the projection $k$-contact locus of $X$ at $p_{0}, \ldots, p_{k}$ and we will denote by $\psi_{k}(X)$, or $\psi_{k}$, its dimension, which is independent of $p_{0}, \ldots, p_{k}$. This will be called the projection $k$ defect.

Remark 3.6. Notice that $\Gamma_{p_{0}, \ldots, p_{k}}$ contains $\Psi_{p_{0}, \ldots, p_{k}}$. Indeed $T_{X, p_{0}, \ldots, p_{k}}$ projects, via $\tau_{k}$, to the tangent space of $X_{k}$ at the point $\tau_{k}\left(p_{0}\right)$, thus it is tangent along the component of the fibre containing $p_{0}$. In particular we get $\gamma_{k} \geq \psi_{k}$. Equality holds if and only if the Gauss map of $X_{k}$ is generically finite to its image, which is equivalent to being birational to its image.

One has $\Psi_{p_{0}, \ldots, p_{i}} \subseteq \Psi_{p_{0}, \ldots, p_{k}}$ for all $i=1, \ldots, k$.

One of the main consequences of Terracini's Lemma is that if $X$ is $k$-defective, then $\psi_{k}>0$, so that $\gamma_{k}$ is also positive.

Other relevant items related to the secant variety $S^{k}(X)$ are the so called entry loci. 
Definition 3.7. Let $x \in S^{k}(X)$ be a point. We define the entry locus $E_{k, x}$ of $x$ with respect to $X$ as the closure of the set

$$
\left\{z \in X: \text { there is } x^{\prime} \in S^{k-1}(X) \text { with } x^{\prime} \neq z \text { and } x \in\left\langle z, x^{\prime}\right\rangle\right\} \text {. }
$$

Alternatively, consider the fibre $F_{x}$ of $p: \mathcal{S}^{k}(X) \rightarrow S^{k}(X)$ over $x$. The entry locus $E_{k, x}$ is the image of $F_{x}$ under the projection $p_{1}: \mathcal{S}^{k}(X) \rightarrow X$ to the first factor.

If $x \in S^{k}(X)$ is a general point, we may denote $E_{k, x}$ simply by $E_{k}$.

Next we can provide interesting information about the $k$-contact loci.

Lemma 3.8. Let $X \subset \mathbb{P}^{r}$ be an irreducible, non-degenerate variety such that $s^{(k)}(X)<r$. If $p_{0}, \ldots, p_{k} \in X$ are general points and $q_{0}, \ldots, q_{k}$ are general points on $\Gamma_{p_{0}, \ldots, p_{k}}$ such that $q_{i}$ specializes to $p_{i}$ for all $i=0, \ldots, k$, then $\Gamma_{p_{0}, \ldots, p_{k}}=\Gamma_{q_{0}, \ldots, q_{k}}$.

Proof. One has $T_{X, q_{i}} \subset T_{S^{k}(X), x}=T_{X, p_{0}, \ldots, p_{k}}$ for all $i=0, \ldots, k$, thus $T_{X, q_{0}, \ldots, q_{k}}=$ $T_{X, p_{0}, \ldots, p_{k}}$. This immediately implies the assertion.

Proposition 3.9. Same hypotheses as in Lemma 3.8 Then:

(i) $\Gamma_{p_{0}, \ldots, p_{k}}$ is smooth at $p_{0}, \ldots, p_{k}$; moreover it is either irreducible or consists of $k+1$ irreducible components of the same dimension $\gamma_{k}$, each containing one of the points $p_{0}, \ldots, p_{k}$ as its general point;

(ii) $f_{i}\left(\Gamma_{k}\right)=f_{i}(X)$ for all $i=1, \ldots, k$;

(iii) $\Pi_{k}=S^{k}\left(\Gamma_{k}\right)$ equals the general Gauss fibre $\Gamma_{S^{k}(X), x}$ of $S^{k}(X)$, whereas $S^{i}\left(\Gamma_{k}\right)$ $\neq \Pi_{k}$ for all $i=1, \ldots, k-1$;

(iv) $t_{k}(X)=\operatorname{dim}\left(\Pi_{k}\right)=k \gamma_{k}+k+\gamma_{k}-f_{k}(X)$.

Proof. Part (i) follows from Lemma 3.8 and from monodromy on the general points $p_{0}, \ldots, p_{k}$ (see [7]).

Let us prove (ii). We assume $\Gamma_{k}=\Gamma_{p_{0}, \ldots, p_{k}}$ is irreducible, otherwise the same argument works. Let $x \in\left\langle p_{0}, \ldots, p_{k}\right\rangle$ be a general point of $S^{k}(X)$. Let also $q_{0}, \ldots, q_{i}$ be general points on $\Gamma_{k}$ and let $y \in\left\langle q_{0}, \ldots, q_{i}\right\rangle$ be a general point of $S^{i}\left(\Gamma_{k}\right)$. By the generality assumption on $p_{0}, \ldots, p_{k}$, also $q_{0}, \ldots, q_{i}$ are general points on $X$, hence $y$ is a general point of $S^{i}(X)$.

Since $q_{0}, \ldots, q_{i}$ are in $\Gamma_{k}$, we have $T_{S^{i}(X), y}=T_{X, q_{0}, \ldots, q_{i}} \subset T_{S^{k}(X), x}$. Moreover, we have an $f_{i}(X)$-dimensional family of $(i+1)$-secant $i$-spaces to $X$ passing through $y$. Let $\left\langle r_{0}, \ldots, r_{i}\right\rangle$ be a general element of such a family, with $r_{0}, \ldots, r_{i} \in X$. Then $T_{X, r_{0}, \ldots, r_{i}}=$ $T_{S^{i}(X), y} \subset T_{S^{k}(X), x}$, which shows that $r_{0}, \ldots, r_{i} \in \Gamma_{k}$. This implies (ii).

Let us prove (iii). We have $S^{k}\left(\Gamma_{p_{0}, \ldots, p_{k}}\right) \subseteq \Pi_{p_{0}, \ldots, p_{k}} \subseteq \Gamma_{S^{k}(X), x}$. Let $y$ be a general point of $\Gamma_{S^{k}(X), x}$, hence $y \in\left\langle q_{0}, \ldots, q_{k}\right\rangle$ with $q_{0}, \ldots, q_{k} \in X$. Since $T_{S^{k}(X), y}=T_{S^{k}(X), x}$, we have $q_{0}, \ldots, q_{k} \in \Gamma_{p_{0}, \ldots, p_{k}}$, thus $y \in S^{k}\left(\Gamma_{p_{0}, \ldots, p_{k}}\right)$. This proves the first assertion. If $S^{i}\left(\Gamma_{k}\right)=\Pi_{k}$ for some $i<k$, then we would have $S^{i}(X)=S^{k}(X)$, contradicting $S^{k}(X) \neq \mathbb{P}^{r}$ (see Proposition 3.2.

Part (iv) easily follows. 
Remark 3.10. It is useful to notice that the $k$-contact loci are responsible for the $k$-fibre defect of $X$. In fact Proposition 3.9 tells us that they have the same $k$-fibre defect of $X$ and through $k+1$ general points of $X$ there is one of them passing.

Moreover, by applying Proposition 3.9 for all positive integers $i<k$, one sees that $S^{i}\left(\Gamma_{i}\right)$ is the linear subspace $\Pi_{i}$, which is also the general Gauss fibre of $S^{i}(X)$, and $t_{i}(X)=i \gamma_{i}+i+\gamma_{i}-f_{i}(X)$. fects.

The next proposition shows an important relation between fibre and projection de-

Proposition 3.11. Let $X \subset \mathbb{P}^{r}$ be an irreducible variety of dimension $n$ such that $s^{(k)}(X)$ $<r$. Then

$$
f_{i}=\psi_{1}+\cdots+\psi_{i}, \quad \forall i \leq k
$$

The same holds if $X$ is reducible but $S^{(k)}(X)$ is pure and $s^{(k)}(X)<r$.

Proof. The proof is by induction on $i$. The case $i=1$ is an immediate consequence of Terracini's Lemma.

Suppose $i>1$. Consider the general $i$-tangential projection $\tau_{i}$ of $X$ from $T_{X, p_{1}, \ldots, p_{i}}$. One has $\operatorname{dim}\left(X_{i}\right)=n-\psi_{i}$ and the general tangent space to $X_{i}$ is the projection of

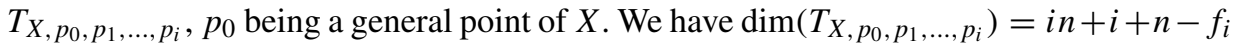
and $\operatorname{dim}\left(T_{X, p_{1}, \ldots, p_{i}}\right)=i n+i-1-f_{i-1}$. Hence $n-\psi_{i}=n-f_{i}+f_{i-1}$, and the assertion follows by induction.

Corollary 3.12. In the above setting, for all $i=1, \ldots, k$, fix general points $p_{1}, \ldots, p_{i}$ in $\Gamma_{k}$. Then the general projection $i$-contact locus of $\Gamma_{k}$ coincides with $\Psi_{i}$. In particular $\psi_{i}\left(\Gamma_{k}\right)=\psi_{i}(X)$.

Proof. Note that $T_{\Gamma_{k}, p_{1}, \ldots, p_{i}} \subseteq T_{X, p_{1}, \ldots, p_{i}} \cap \Pi_{k}$. Moreover $T_{X, p_{1}, \ldots, p_{i}}$ does not contain $\Gamma_{k}$, otherwise it would contain the whole of $X$, since $p_{0} \in \Gamma_{k}$ is a general point of $X$. Therefore it makes sense to consider the restriction to $\Gamma_{k}$ of the $i$-tangential projection $\tau_{X, i}$, which factors through the $i$-tangential projection $\tau_{\Gamma_{k}, i}$. This implies that $\psi_{i}\left(\Gamma_{k}\right) \leq \psi_{i}(X)$. By Propositions 3.11 and 3.9 (ii), equality has to hold for all $i=1, \ldots, k$ and the assertion follows.

Useful information about the entry loci is provided by the following:

Proposition 3.13. Let $X \subset \mathbb{P}^{r}$ be an irreducible variety with $s^{(k)}(X)<r$. Then $E_{k}$ is pure of dimension $\psi_{k}$. The same holds if $X$ is reducible and $S^{k-1}(X)$ and $S^{k}(X)$ are pure.

Proof. Let $x \in S^{k}(X)$ be a general point. The fibre $F_{x}$ of $p: \mathcal{S}^{k}(X) \rightarrow S^{k}(X)$ over $x$ is pure of dimension $f_{k}$. The projection to the first factor yields a dominant map $q$ : $F_{x} \rightarrow E_{k, x}$. Let $z$ be a general point in a component of $E_{k, x}$, and let $F_{x, z}$ be the fibre of $q$ over $z$. Let $\xi=\left(z, p_{1}, \ldots, p_{k}, x\right)$ be a general point in a component $Z$ of $F_{x, z}$. Note that $\left\langle p_{1}, \ldots, p_{k}\right\rangle$ is a $(k-1)$-space intersecting the line $\langle x, z\rangle$ at a point $p$. By Proposition 3.2, the point $p$ does not depend on $\xi$. Moreover one sees that, for $x$ and $z$ general, $p$ is a general point in $S^{k-1}(X)$. Hence we have a map

$$
Z \rightarrow \mathcal{S}^{k-1}(X), \quad \xi \mapsto\left(p_{1}, \ldots, p_{k}, p\right),
$$


which is birational to its image, and this, in turn, is a component of the fibre of $\mathcal{S}^{k-1}(X)$ $\rightarrow S^{k-1}(X)$ over $p$. This shows that any component $Z$ of $F_{x, z}$ has dimension $f_{k-1}$. Hence any component of $E_{k, x}$ has dimension $f_{k}-f_{k-1}=\psi_{k}$ (see Proposition 3.11.

A different proof of Proposition 3.13 follows from Proposition 2.2 of [16], which asserts that $\Psi_{k}$ can be seen as a degeneration of $E_{k}$.

Remark 3.14. Terracini's Lemma implies that for $x \in S^{k}(X)$ general and for a general point $z \in E_{k, x}$, one has $T_{X, z} \subseteq T_{S^{k}(X), x}$. Hence $E_{k, x}$ is contained in the tangential $k$ contact locus $\Gamma_{p_{0}, \ldots, p_{k}}$ for all $p_{0}, \ldots, p_{k} \in E_{k, x}$ which are smooth points of $X$ and such that $x \in\left\langle p_{0}, \ldots, p_{k}\right\rangle$. Again we deduce $\psi_{k} \leq \gamma_{k}$.

We finish by recalling the following well known subadditivity theorem by PalatiniZak, whose proof is an application of the previous results (see [27, Chapter V, Proposition 1.7 and Theorem 1.8]). One more piece of notation before that. Let $X \subset \mathbb{P}^{r}$ be an irreducible, non-degenerate variety. We set

$$
k_{0}:=k_{0}(X)=\min \left\{k \in \mathbb{N}: S^{k}(X)=\mathbb{P}^{r}\right\} .
$$

Theorem 3.15. Let $X \subset \mathbb{P}^{r}$ be a smooth, irreducible, non-degenerate variety of dimension $n$. Then

(i) $\psi_{1} \leq \cdots \leq \psi_{k_{0}} \leq n$;

(ii) $\psi_{k} \geq \psi_{k-1}+\psi_{1}$ for all $k \leq k_{0}$.

From Theorem 3.15 one deduces

$$
\psi_{k} \geq k \psi_{1}, \quad \forall k \leq k_{0} .
$$

Definition 3.16 (see [27, Chapter VI, Proposition 1.2]). A smooth, irreducible, nondegenerate variety $X \subset \mathbb{P}^{r}$ of dimension $n$ with $\psi_{1}>0$ is called a Scorza variety if equality holds in (3.1) and in addition $k_{0}=\left[n / \psi_{1}\right]$.

The classification of Scorza varieties is contained in [27, Chapter VI].

Remark 3.17. If $X$ is smooth, and the general entry locus $E_{1}$ is a quadric, then $E_{1}$ is smooth (see [11, pp. 964-965]). To the best of our knowledge, there is no argument for the smoothness of the general entry locus $E_{k}$, though in [27, p. 123], this is claimed to be a consequence of "usual general position arguments" which we are unable to understand. The classification of Scorza varieties in [27] seems to depend on this assertion, which is however false, in its full generality, as the following example shows.

Example 3.18. Consider the scroll $X=S(1, h) \subset \mathbb{P}^{h+2}$ with $h \geq 4$. Let $L$ be the line directrix of $X$. For all $k \geq 1, S^{k}(X)$ is the cone with vertex $L$ over $S^{k}(Y)$, with $Y$ a rational normal curve in an $h$-space $\Pi^{\prime}$ which is skew with $L$. Therefore

$$
s^{(k)}(X)=2 k+3<h+2
$$

as soon as $k<(h-1) / 2$, and $X$ is $k$-defective if $k \geq 2$. In this case it is not difficult to see that $E_{k}$ is formed by $k+1$ general rulings of $X$ plus the line $L$. 


\section{Zak's theorem on tangencies}

Zak's theorem on tangencies is a basic tool for the study of projective varieties and their secant varieties (see [26], [27]). It says that if a linear space $L$ in $\mathbb{P}^{r}$ is tangent to a smooth, pure variety $X \subset \mathbb{P}^{r}$ along a subvariety $Y$, then

$$
\operatorname{dim}(L) \geq \operatorname{dim}(X)+\operatorname{dim}(Y) .
$$

Zak's original formulation in [26] is more general, inasmuch as it also applies to singular varieties $X$ : in this case the dimension of the singular locus of $X$ enters into play. On the other hand, as proved in Zak's book [27], formula (4.1] also works for singular varieties $X$, provided one takes the right definition of tangency at singular points. Let us recall this definition.

Definition 4.1. Let $X$ be a variety, $L$ a linear subspace of $\mathbb{P}^{r}$, and $Y$ a subvariety of $X$ contained in $L$. One says that $L$ is $J$-tangent to $X$ along $Y$ if the following holds. Let $\left\{\left(q_{0}(t), q_{1}(t)\right)\right\}_{t \in \Delta}$ be any analytic curve in $X \times Y$ parametrized by the unitary disc $\Delta$ and such that:

(i) $q_{0}(t) \notin L$ for any $t \in \Delta-\{0\}$;

(ii) $q_{0}(0) \in L$.

Then the flat limit of the line $\left\langle q_{0}(t), q_{1}(t)\right\rangle$ lies in $L$.

Remark 4.2. In point (ii) of Definition 4.1 one may equivalently ask that $q_{0}(0) \in Y$. Indeed, if $q_{0}(0) \in L$ but not in $Y$, then it is clear that the flat limit of the line $\left\langle q_{0}(t), q_{1}(t)\right\rangle$ lies in $L$, since $q_{1}(0) \in Y$ and $q_{0}(0) \neq q_{1}(0)$.

If $L$ is $J$-tangent to $X$ along $Y$, then it is also $J$-tangent to $X$ along any subvariety $Z$ contained in $Y$.

If $L$ is $J$-tangent to $X$ along $Y$, then $L$ is $J$-tangent to any irreducible component of $X$ along any irreducible component of $Y$.

If $L$ is $J$-tangent to $X$ along $Y$, and $Z$ is a subvariety of $X$ containing $Y$ but not contained in $L$, then $L$ is also $J$-tangent to $Z$ along $Y$.

If $X$ is smooth along $Y$, being $J$-tangent is equivalent to the condition that $L$ contains the tangent space $T_{X, y}$ to $X$ at a general point $y \in Y$. If $X$ is singular at some point $y \in Y$, $J$-tangency imposes further restrictions on $L$.

Example 4.3. If $X \subset \mathbb{P}^{r}$ is a non-degenerate cone with vertex $v$, then no proper subspace of $\mathbb{P}^{r}$ can be $J$-tangent to $X$ along a subvariety containing $v$.

The notion of $J$-tangency provides a suitable setting for a general formulation of Zak's theorem on tangencies valid for singular varieties.

Theorem 4.4 (Zak's theorem on tangencies). Let $X \subset \mathbb{P}^{r}$ be a variety. If a linear space $L$ of $\mathbb{P}^{r}$ is $J$-tangent to $X$ along a subvariety $Y$, then (4.1) holds.

It is well known that this theorem is sharp. 
Example 4.5. There are smooth surfaces $X$ in $\mathbb{P}^{4}$ with a hyperplane $H$ tangent to $X$ along a curve. An example is the projection $X$ to $\mathbb{P}^{4}$ of the Veronese surface $V_{2,2}$ in $\mathbb{P}^{5}$ from a general point $p \in \mathbb{P}^{5}$.

In fact, the Veronese surface has a 2-dimensional system of conics and there is a hyperplane tangent to $V_{2,2}$ along each conic. Hence, there is a 1-dimensional system of these tangent hyperplanes passing through the centre of projection $p$. Such hyperplanes project down to $\mathbb{P}^{4}$ to hyperplanes of $\mathbb{P}^{4}$ which are tangent to $X$ along the corresponding conics.

To be more specific, let $Y$ be any conic on $X$. Consider the pencil of hyperplanes containing $Y$. This pencil cuts out on $X$, off $Y$, a pencil of conics having a base point $y$. There is a hyperplane tangent to $X$ along $Y$ if and only if $y \in Y$.

Since the map associating $y$ to $Y$ is a projective transformation $\omega:\left(\mathbb{P}^{2}\right)^{*} \rightarrow \mathbb{P}^{2}$, the locus of points $y \in X$ belonging to the corresponding conic $Y$ describes a conic in $\mathbb{P}^{2}$ and therefore a rational normal quartic on $X$.

Although sharp, Zak's theorem can be improved, as we shall see. To do this, we first have to extend the notion of $J$-tangency. We will do this in the next section.

\section{The notion of $J_{k}$-tangency}

In order to improve Zak's theorem on tangencies, we need to extend the concept of $J$ tangency.

Definition 5.1. Let $X, L$ and $Y$ be as in Definition 4.1 Let $k$ be a positive integer such that $\operatorname{dim}(\langle Y\rangle) \geq k-1$. One says that $L$ is $J_{k}$-tangent to $X$ along $Y$ if the following holds. Let $\left\{\left(q_{0}(t), q_{1}(t), \ldots, q_{s}(t)\right)\right\}_{t \in \Delta}$, with $1 \leq s \leq k$, be any analytic curve in $X \times Y^{s}$ parametrized by the unitary disc $\Delta$ and such that:

(i) $\left(q_{0}(t), \ldots, q_{s}(t)\right)$ are linearly independent for any $t \in \Delta-\{0\}$;

(ii) $q_{0}(t) \notin L$ for any $t \in \Delta-\{0\}$;

(iii) $q_{0}(0) \in L$.

Then the flat limit of the $s$-space $\left\langle q_{0}(t), q_{1}(t), \ldots, q_{s}(t)\right\rangle$ as $t \rightarrow 0$ lies in $L$.

Remark 5.2. Of course $J_{1}$-tangency is $J$-tangency. Remark 4.2 can be repeated verbatim replacing $J$-tangency with $J_{k}$-tangency.

In particular, in point (iii) of Definition 5.1 one may equivalently ask that $q_{0}(0) \in$ $J\left(Y^{s}\right) \cap X$. Indeed, if $q_{0}(0)$ lies in $L$ but not on $J\left(Y^{s}\right) \cap X$, then the flat limit of the $s$-space $\left\langle q_{0}(t), q_{1}(t), \ldots, q_{s}(t)\right\rangle$ as $t \rightarrow 0$ lies in $L$, since it is spanned by the flat limit $\Pi$ of the $(s-1)$-space $\left\langle q_{1}(t), \ldots, q_{s}(t)\right\rangle$, which lies in $L$, and by $q_{0}(0)$ which lies in $L$ and not on $\Pi$.

Finally $J_{k}$-tangency implies $J_{h}$-tangency for all $h<k$, but the converse does not hold.

Example 5.3. Let us go back to Example 4.5, from which we keep the notation. Consider a hyperplane $H$ tangent, and therefore $J$-tangent, to $X$ along a conic $Y$. Let us show that $H$ is not $J_{2}$-tangent to $X$ along $Y$. Let $\Pi=\langle Y\rangle$ and let $y \in Y$ be as in Example 4.5 
Note that all hyperplanes through $\Pi$ cut on $X$ a curve of the form $Y+Z$, with $Z$ a conic through $y$. This shows that $\Pi=T_{X, y}$. Take a general conic $Z$ on $X$ through $y$. Set $\Pi^{\prime}=\langle Z\rangle$. Then $\Pi \cap \Pi^{\prime}$ is a line $\ell$ which is the tangent line to $Z$ at $y$ and is also a general line in $\Pi$ through $y$. Let $x$ be the intersection of $\ell$ with $Y$ other than $y$. Consider an analytic parametrization $p(t)$ of $Z$ around $y$, so that $p(0)=y \in Y$, and consider the analytic curve $(p(t), x, y)$ in $X \times Y^{2}$. Then the 2 -space $\langle p(t), x, y\rangle=\Pi^{\prime}$ does not depend on $t$, and therefore its limit is $\Pi^{\prime}$, which does not lie in $H$.

The notion of $J_{k}$-tangency will play a crucial role below. Let us add a couple of related definitions.

Definition 5.4. Let $X \subset \mathbb{P}^{r}$ be variety, $Y$ a subvariety of $X$ and let $k$ be a positive integer. We say that $X$ is $k$-smooth along $Y$ if $X$ is smooth along $Y$ and any subscheme $Z$ of $X$ of finite length $s \leq k+1$ supported at $Y$ spans a linear space of dimension $s-1$. We say that $X$ is $k$-smooth if it is $k$-smooth along $X$.

Definition 5.5. Let $X \subset \mathbb{P}^{r}$ be a variety such that $s^{(k)}(X)<r$. We will say that $X$ enjoys the $R_{k}$-property (or briefly that $X$ is an $R_{k}$-variety) if the following holds. For any $i=1, \ldots, k$ and general points $p_{0}, \ldots, p_{i}$, taken in different components of $X$ if $X$ is reducible, the general hyperplane tangent to $X$ at $p_{0}, \ldots, p_{i}$ is $J_{i}$-tangent to $X$ along $\Gamma_{p_{0}, \ldots, p_{i}}$.

Remark 5.6. (a) The notion of $k$-smoothness is hereditary, i.e., if $X$ is $k$-smooth along $Y$, then $X$ is $k$-smooth along any subvariety $Z$ of $Y$.

(b) The notion of $k$-smoothness coincides with $\mathcal{O}_{X}(1)$ being $k$-very ample (see [3]). It can also be rephrased as follows: $X$ is $k$-smooth along $Y$ if there is no linear space $L$ of dimension $s<k$ containing a subscheme $Z$ of $X$ of finite length $\ell \geq s+2$ supported at $Y$.

Note that $k$-smoothness is a rather rigid notion. For example a smooth variety containing a line is not $k$-smooth for any $k \geq 2$. On the other hand, if $X$ is smooth, its $d$-tuple Veronese embedding, with $d \geq k+1$, is $k$-smooth.

Next we will show a relationship between the notions of $k$-smoothness and $J_{k}$-tangency.

Lemma 5.7. Let $X \subset \mathbb{P}^{r}$ be a variety, and $Y$ a subvariety of $X$. Assume that $X$ is smooth along $Y$ and let $L$ be a linear space tangent to $X$ along $Y$. Let $\left\{\left(q_{0}(t), q_{1}(t), \ldots, q_{s}(t)\right)\right\}_{t \in \Delta}$ be an analytic curve in $X \times Y^{s}$ parametrized by the unitary disc $\Delta$ such that $q_{0}(t), q_{1}(t), \ldots, q_{s}(t)$ are distinct for $t \in \Delta-\{0\}$ and $q_{0}(0) \in$ L. Let $Z_{0}$ be the flat limit of the reduced 0-dimensional scheme corresponding to the 0-cycle $Z_{t}=q_{0}(t)+q_{1}(t)+\cdots+q_{s}(t)$. Then $Z_{0}$ is contained in $L$.

Proof. Let $Z$ be the limit of the 0-dimensional scheme corresponding to the 0-cycle $q_{1}(t)+\cdots+q_{s}(t)$. Note that $Z$ sits in $Y$ and therefore in $L$. The degrees of $Z$ and $Z_{0}$ differ by 1 . If $Z$ and $Z_{0}$ do not share the same support, then the assertion is clear. If $Z$ and $Z_{0}$ have the same support, then $q_{0}(0) \in Y$. Moreover the ideal sheaves of $Z$ and 
$Z_{0}$ behave as follows: for any point $p$ of the common support one has $\mathscr{I}_{Z_{0}, p} \subseteq \mathscr{I}_{Z, p}$ and the inclusion is strict only at one point $q$, where $\mathscr{I}_{Z, q} / \mathscr{I}_{Z_{0}, q}=\mathbb{C}$. This corresponds to a single condition imposed on functions in $\mathscr{I}_{Z, q}$ in order to have functions in $\mathscr{I}_{Z_{0}, q}$. This is clearly a tangential condition, i.e. the functions in $\mathscr{I}_{Z, q}$, in order to be in $\mathscr{I}_{Z_{0}, q}$, are required to be annihilated by the differential operator corresponding to the tangent vector to the branch of the curve $\left\{q_{0}(t)\right\}_{t \in \Delta}$ at $t=0$. Since $L$ is tangent to $X$ along $Y$, this condition is satisfied by the equations of $L$ as well, proving the assertion.

Proposition 5.8. Let $X \subset \mathbb{P}^{r}$ be a variety, and $Y$ a subvariety of $X$. Assume that $X$ is $k$-smooth along $Y$. Then a linear space $L$ is $J_{k}$-tangent to $X$ along $Y$ if and only if it is tangent to $X$ along $Y$.

Proof. Assume $L$ is tangent to $X$ along $Y$. Let $\left\{\left(q_{0}(t), q_{1}(t), \ldots, q_{s}(t)\right)\right\}_{t \in \Delta}$, with $1 \leq$ $s \leq k$, be any analytic curve in $X \times Y^{s}$ as in Definition 5.1

Suppose the limit $\Pi_{0}$ of the $s$-space $\Pi_{t}=\left\langle q_{0}(t), q_{1}(t), \ldots, q_{s}(t)\right\rangle$ does not lie in $L$. Then $\Pi_{0} \cap L$ would be a linear space of dimension $t<s$ containing the scheme $Z_{0}$ (see Lemma 5.7]. This contradicts the $k$-smoothness assumption.

Remark 5.9. The converse of Proposition 5.8 does not hold, i.e. there are varieties which are $k$-regular but not $k$-smooth. For example, the $\operatorname{Segre}$ variety $\operatorname{Seg}(n, n)$, with $n \geq 3$, is not 2-smooth because it contains lines, hence also triples of collinear points, but it is an $R_{n-1}$-variety (see Example 5.14 below).

Next we point out a couple of easy lemmata.

Lemma 5.10. Let $X, Y, k$ be as in Definition 5.4. Let $L$ be a linear space which is $J_{k}$ tangent to $X$ along $Y$. Fix a point $p \notin S^{k}(X)$ and let $\pi$ be the projection from $p$. Then $L^{\prime}=\pi(L)$ is $J_{k}$-tangent to $X^{\prime}=\pi(X)$ along $Y^{\prime}=\pi(Y)$.

Proof. Let $\left\{\left(q_{0}(t), q_{1}(t), \ldots, q_{s}(t)\right)\right\}_{t \in \Delta}$, with $1 \leq s \leq k$, be an analytic curve as in Definition 5.1. Then the limit $\Pi$ of the $s$-space $\left\langle q_{0}(t), q_{1}(t), \ldots, q_{s}(t)\right\rangle$ as $t \rightarrow 0$ lies in $L$ and does not contain $p$.

Consider the curve $\left\{\left(\pi\left(q_{0}(t)\right), \pi\left(q_{1}(t)\right), \ldots, \pi\left(q_{s}(t)\right)\right)\right\}_{t \in \Delta}$ in $X^{\prime} \times Y^{\prime s}$. It enjoys properties (i)-(iii) of Definition 5.1 with $Y, L$ replaced by $Y^{\prime}, L^{\prime}$. The limit of the $s$-space $\left\langle\pi\left(q_{0}(t)\right), \pi\left(q_{1}(t)\right), \ldots, \pi\left(q_{s}(t)\right)\right\rangle$ is the projection of $\Pi$ from $p$, hence it is an $s$-space contained in $L^{\prime}$. On the other hand, any curve in $X^{\prime} \times Y^{\prime s}$ enjoying properties (i)-(iii) of Definition 5.1 with $Y, L$ replaced by $Y^{\prime}, L^{\prime}$ can be obtained in this way.

Lemma 5.11. Let $X, Y, k$ be as in Definition 5.4 with $k \geq 2$. Let L be a linear space which is $J_{k}$-tangent to $X$ along $Y$. Let $p$ be a point on $Y$ and let $\pi$ be the projection from $p$. Then $L^{\prime}=\overline{\pi(L)}$ is $J_{k-1}$-tangent to $X^{\prime}=\overline{\pi(X)}$ along $Y^{\prime}=\overline{\pi(Y)}$.

Proof. Suppose the assertion is not true. Then we can find an analytic curve $\left\{\left(q_{0}(t), \ldots\right.\right.$, $\left.\left.q_{s}(t)\right)\right\}_{t \in \Delta}$, with $1 \leq s \leq k-1$, in $X^{\prime} \times Y^{\prime s}$ satisfying (i)-(iii) of Definition 5.1 with $Y, L$ replaced by $Y^{\prime}, L^{\prime}$, and such that the limit $\Pi^{\prime}$ of the $s$-space $\left\langle q_{0}(t), q_{1}(t), \ldots, q_{s}(t)\right\rangle$ as $t \rightarrow 0$ does not lie in $L^{\prime}$. By slightly perturbing this curve if necessary, we may assume that it can be lifted to an analytic curve $\left\{\left(p_{0}(t), \ldots, p_{s}(t)\right)\right\}_{t \in \Delta}$ in $X \times Y^{s}$. Consider then 
the analytic curve $\left\{\left(p_{0}(t), \ldots, p_{s}(t), p_{s+1}(t)\right)\right\}_{t \in \Delta}$ in $X \times Y^{s+1}$, with $p_{s+1}(t)$ independent of $t$ and equal to $p$. This curve satisfies (i)-(iii) of Definition 5.1 and therefore the limit $\Pi$ of the $(s+1)$-space $\left\langle p_{0}(t), \ldots, p_{s}(t), p\right\rangle$ as $t \rightarrow 0$ lies in $L$. But then its projection from $p$, which is $\Pi^{\prime}$, should lie in $L^{\prime}$, a contradiction.

Remark 5.12. As in Lemma 5.10, one has the following. Let $X$ be an $R_{k}$-variety [resp. a $k$-smooth variety] and fix a point $p \notin S^{k}(X)$. Then the image of the projection $\pi$ of $X$ from $p$ is again an $R_{k}$-variety [resp. a $k$-smooth variety].

Similar considerations hold for Lemma 5.11.

The following provides a simple criterion for the $R_{k}$-property.

Proposition 5.13. Let $X \subset \mathbb{P}^{r}$ be an irreducible, non-degenerate variety such that ${ }^{(k)}(X)$ $<r$. Assume that $\gamma_{i}=\psi_{i}$ for all $i=1, \ldots, k$. Assume moreover that the intersection of the indeterminacy loci of all tangential projections $\tau_{p_{0}, \ldots, p_{i}}$ is empty for $p_{0}, \ldots, p_{i}$ being general points in $X$. Then $X$ is an $R_{k}$-variety.

Proof. Fix any $i=1, \ldots, k$ and general points $p_{0}, \ldots, p_{i}$. Consider the $i$-tangential projection $\tau_{i}$ of $X$ from $T_{X, p_{1}, \ldots, p_{i}}$ and set $p=\tau_{i}\left(p_{0}\right)$. Note that $\gamma_{i}=\psi_{i}$ implies that $\Psi_{p_{0}, \ldots, p_{i}}$ is the irreducible component of $\Gamma_{p_{0}, \ldots, p_{i}}$ containing $p_{0}$. Assume $\Gamma_{i}=\Gamma_{p_{0}, \ldots, p_{i}}$ is irreducible. The argument in the reducible case is the same, and can be left to the reader. Thus $p$ is the image via $\tau_{i}$ of $\Gamma_{i}=\Gamma_{p_{0}, \ldots, p_{i}}$. Consider a general hyperplane $H$ tangent to $X$ along $\Gamma_{i}$. Let $H^{\prime}$ be the image of $H$ via $\tau_{i}$, tangent to $X_{i}$ at $p$, which is a smooth point of $X_{i}$.

Take a curve $\left\{\left(q_{0}(t), q_{1}(t), \ldots, q_{s}(t)\right)\right\}_{t \in \Delta}$, with $1 \leq s \leq i$, in $X \times \Gamma_{i}^{s}$ satisfying (i)-(iii) of Definition 5.1 with $Y=\Gamma_{i}$. Choose $p_{0}, \ldots, p_{i}$ on $\Gamma_{i}$ in such a way that none of the points $q_{0}(t), q_{1}(t), \ldots, q_{s}(t)$, for $t$ general in $\Delta$, sits in the indeterminacy locus of $\tau_{i}$. Then the projection via $\tau_{i}$ of the limit $\Pi$ of the $s$-space $\left\langle q_{0}(t), q_{1}(t), \ldots, q_{s}(t)\right\rangle$ as $t \rightarrow 0$ sits in $T_{X_{i}, p}$, which in turn sits in $H^{\prime}$. This implies that $\Pi$ sits in $H$, proving the assertion.

Example 5.14. The previous proposition implies that the following varieties are $R_{k}$ varieties:

(i) the $(k+1)$-dimensional Veronese variety $V_{2, k+1}$ in $\mathbb{P}^{k(k+3) / 2}$;

(ii) the $2(k+1)$-dimensional Segre variety $\operatorname{Seg}(k+1, k+1)$ in $\mathbb{P}^{k^{2}+4 k+3}$;

(iii) the $4(k+1)$-dimensional Grassmann variety $\mathbb{G}(1,2 k+3)$ in $\mathbb{P}^{\left(\begin{array}{c}2 k+4 \\ 2\end{array}\right)-1}$.

Indeed, the $i$-contact locus of $V_{2, k+1}$ is the Veronese image of a general linear subspace of dimension $i$. This is also the fibre of the general $i$-tangential projection of $V_{2, k+1}$.

Similarly the $i$-contact locus of $\operatorname{Seg}(k+1, k+1)$ is a subvariety of type $\operatorname{Seg}(i, i)$, which is also the fibre of the general $i$-tangential projection of $\operatorname{Seg}(k+1, k+1)$.

Finally the $i$-contact locus of $\mathbb{G}(1,2 k+3)$ is a subvariety of type $\mathbb{G}(1,2 i+1)$, which is also the fibre of the general $i$-tangential projection of $\operatorname{Seg}(k+1, k+1)$.

The condition about the indeterminacy loci is easily seen to be satisfied in all these cases. 


\section{An extension of Zak's theorem on tangencies}

The notion of $J_{k}$-tangency plays a basic role in the following extension of Zak's theorem on tangencies.

Theorem 6.1. Let $X \subset \mathbb{P}^{r}$ be a non-degenerate variety and let $L \neq \mathbb{P}^{r}$ be a proper linear subspace of $\mathbb{P}^{r}$ which is $J_{k}$-tangent to $X$ along a pure subvariety $Y$. Then

$$
\operatorname{dim}(L) \geq \operatorname{dim}(X)+\operatorname{dim}\left(J^{k}(Y)\right) \geq \operatorname{dim}(X)+\operatorname{dim}\left(S^{k-1}(Y)\right) .
$$

In particular, 6.1) holds when $L$ is tangent to $X$ along $Y$ and $X$ is $k$-smooth along $Y$.

Proof. The proof follows Zak's original argument.

By definition we have $\operatorname{dim}(\langle Y\rangle) \geq k-1$. Fix $k$ independent points $p_{1}, \ldots, p_{k} \in Y$ such that $N:=\left\langle p_{1}, \ldots, p_{k}\right\rangle$ lies in a component $Z \subseteq J^{k}(Y)$ of maximal dimension. Fix $p_{0} \in X$ general, so that $p_{0} \notin L$. Set $M:=\left\langle p_{0}, p_{1}, \ldots, p_{k}\right\rangle$, so that $\operatorname{dim}(M)=k$.

We may assume that $M$ is not contained in $X$, otherwise $X$ would be a cone with vertex $N$, contradicting the fact that $L$ is $J_{k}$-tangent to $X$ along $Y$ (see Example 4.3.

Pick a general point $x \in M-X$ and let $f: X \times J^{k}(Y) \rightarrow L \times L$ be the morphism which is the inclusion on the second coordinate and the projection $\pi$ from a general linear space $\Pi$ of dimension $r-\operatorname{dim}(L)-1$ containing $x$ on the first coordinate. By the generality assumption one has $\Pi \cap L=\Pi \cap X=\emptyset$.

We claim that $f$ is finite. Suppose in fact $C$ is a curve mapping to a point via $f$. Its projection on $X$ would be a curve $C^{\prime}$, since $f$ is injective on the second coordinate. Moreover $\pi\left(C^{\prime}\right)=y$ would be a point, and therefore $C^{\prime} \subset\langle y, \Pi\rangle$. But then $X \cap \Pi \supseteq$ $C^{\prime} \cap \Pi \neq \emptyset$, a contradiction.

Now consider the component $X_{0}$ of $X$ passing through $p_{0}$ and restrict the map $f$ to $X_{0} \times Z$. If $\operatorname{dim}(L)<\operatorname{dim}(X)+\operatorname{dim}\left(J^{k}(Y)\right)=\operatorname{dim}\left(X_{0}\right)+\operatorname{dim}(Z)$, Fulton-Hansen's connectedness theorem (see [12]) implies that the inverse image of the diagonal $D$ of $L \times L$ is connected.

Notice that $\pi\left(p_{0}\right)=y \in N$. Thus $f\left(p_{0}, y\right)=(y, y)$. Since also $(x, x)$, with $x \in$ $X \cap J^{k}(Y)$, belongs to the inverse image of $D$, Fulton-Hansen's theorem implies that there is a curve $\left\{\left(q_{0}(t), q_{1}(t), \ldots, q_{k}(t)\right)\right\}_{t \in \Delta}$ in $X_{0} \times Y^{k}$ such that:

(i) $q_{1}(t), \ldots, q_{k}(t)$ are linearly independent for any $t \in \Delta-\{0\}$;

(ii) $q_{0}(t)$ is a general point in $X_{0}$ for $t \in \Delta-\{0\}$.

(iii) $\left.\pi\left(q_{0}(t)\right) \in Q_{t}:=\left\langle q_{1}(t), \ldots, q_{k}(t)\right)\right\rangle \subset Z$ for all $t \in \Delta-\{0\}$;

(iv) $q_{0}(0) \in J^{k}(Y) \cap X$.

The $J_{k}$-tangency hypothesis implies that the limit $P$ of $P_{t}:=\left\langle q_{0}(t), q_{1}(t), \ldots, q_{k}(t)\right\rangle$ as $t \rightarrow 0$ lies in $L$. On the other hand, since $\pi\left(q_{0}(t)\right) \in Q_{t}$ for all $t \in \Delta-\{0\}$, we have $\Pi \cap P_{t} \neq \emptyset$ for all $t \in \Delta-\{0\}$, and therefore $\Pi \cap P \neq \emptyset$. Thus $L \cap \Pi \neq \emptyset$, a contradiction.

Remark 6.2. Theorem 6.1 is sharp. The Veronese surface $V_{2,2}$ has hyperplanes $L$ which are $J_{2}$-tangent along a conic $Y$, because $V_{2,2}$ is 2 -smooth. In that case $S^{1}(Y)$ is a plane and in 6.1) equality holds. This extends to higher Veronese varieties $V_{2, r}, r \geq 3$. 
The $J_{k}$-tangency hypothesis is essential in Theorem 6.1. Indeed, for a general projection $X$ of $V_{2,2}$ in $\mathbb{P}^{4}$, the inequality 6.1) does not hold, but $X$ is not 2-smooth (since it has trisecant lines, see Example 4.5.

\section{An extension of Zak's theorem on linear normality}

A striking consequence of the theorem on tangencies is the famous:

Theorem 7.1 (Zak's theorem on linear normality). Let $X \subset \mathbb{P}^{r}$ be a smooth, irreducible, non-degenerate variety of dimension $n$. Then

$$
s(X) \geq \min \{r, 3 n / 2+1\} .
$$

The reason for the name of the theorem is that it gives a positive answer to the following conjecture by Hartshorne (see [14]):

Conjecture 7.2 (Hartshorne's conjecture on linear normality). Let $X \subset \mathbb{P}^{r}$ be a smooth, irreducible, non-degenerate variety of dimension $n$. If $3 n>2(r-1)$ then $X$ is linearly normal.

In this section we want to extend Zak's Theorem 7.1 by giving a lower bound on $s^{(k)}(X)$ under suitable assumptions on the variety $X \subset \mathbb{P}^{r}$.

Let us prove the following key lemma:

Lemma 7.3. Let $X \subset \mathbb{P}^{r}$ be a non-degenerate variety such that $s^{(k)}(X)<r$. Assume $X$ is an $R_{k}$-variety (or $X$ is $k$-smooth). Then

$$
2 f_{k}(X) \leq k n \text {. }
$$

Moreover, if equality holds, then:

$$
\begin{aligned}
& \text { (i) } \gamma_{i}=\psi_{i}=\text { if } \text { for all } i=1, \ldots, k \text {, } \\
& \text { (ii) } f_{i}=\frac{i(i+1)}{2} f \quad \text { for all } i=1, \ldots, k \text {, } \\
& \text { (iii) } n=(k+1) f \text {, }
\end{aligned}
$$

where, as usual, we set $f=f_{1}$.

Proof. After projecting generically and applying Lemma 5.10, we may reduce ourselves to the case $r=n k+n+k-f_{k}+1$.

Consider the general tangential $i$-contact locus $\Gamma_{i}=\Gamma_{p_{0}, \ldots, p_{i}}$ for all $i=1, \ldots, k$. By the hypothesis, the general hyperplane $H_{i}$ tangent to $X$ at $p_{0}, \ldots, p_{i-1}$ is $J_{i-1}$-tangent to $X$ along $\Gamma_{i-1}$. Moreover it does not contain $\Gamma_{i}$. Hence it is also $J_{i-1}$-tangent to $\Gamma_{i}$ along $\Gamma_{i-1}$. Since $S^{i-1}\left(\Gamma_{i}\right)$ does not fill up $\Pi_{i}$ (see Proposition 3.9), after projecting from a general point of $H_{i} \cap \Pi_{i}$, by Lemma 5.10 we find a linear space of dimension $\operatorname{dim}\left(H_{i} \cap \Pi_{i}\right)-1=\operatorname{dim}\left(\Pi_{i}\right)-2$, which is $J_{i-1}$-tangent to the projection of $\Gamma_{i}$ along $\Gamma_{i-1}$. Then, by Proposition 3.2 and Theorem 6.1. we deduce that

$$
\operatorname{dim}\left(\Pi_{i}\right)-2 \geq \operatorname{dim}\left(\Gamma_{i}\right)+\operatorname{dim}\left(S^{i-2}\left(\Gamma_{i-1}\right)\right) .
$$


Using Proposition 3.9, this inequality translates into

$$
i \gamma_{i} \geq(i-1) \gamma_{i-1}+f_{i}-f_{i-2}
$$

for all $i=2, \ldots, k$, where we put $f_{0}=0$. Adding these relations up, and taking into account that $\gamma_{1} \geq f_{1}=\psi_{1}$, we deduce

$$
k \gamma_{k} \geq f_{k}+f_{k-1}
$$

Now notice that there exists a hyperplane $H$ in $\mathbb{P}^{r}$ which is $J_{k}$-tangent to $X$ along $\Gamma_{k}$. With the same argument as above, we find

$$
n k \geq k \gamma_{k}+f_{k}-f_{k-1}
$$

By taking into account (7.4), 7.1) follows.

Suppose $2 f_{k}=k n$. Then equality holds in 7.3 for all $i=1, \ldots, k$. In particular we get $\gamma_{1}=\psi_{1}=f$. Thus (i) of 7.2 holds for $i=1$. Assume $i \geq 2$ and proceed by induction.

By Proposition 3.11, we know that $\gamma_{i} \geq \psi_{i}=f_{i}-f_{i-1}$. This, together with 7.3 (where we must have an equality), yields

$$
(i-1) \gamma_{i} \leq(i-1) \gamma_{i-1}+f_{i-1}-f_{i-2}
$$

so that, by induction,

$$
(i-1) \gamma_{i} \leq(i-1) \gamma_{i-1}+\psi_{i-1}=i \gamma_{i-1}=i(i-1) f,
$$

thus if $\geq \gamma_{i}$. On the other hand, by induction and subadditivity (see Theorem 3.15), we have

$$
\gamma_{i} \geq \psi_{i} \geq \psi_{i-1}+\psi_{1}=\gamma_{i-1}+f=i f
$$

so that if $=\gamma_{i}$. This proves (i), and (ii) immediately follows; moreover $k n=2 f_{k}=$ $k(k-1) f$ and also (iii) is proved.

Example 7.4. There are examples of $R_{k}$-varieties $X$ for which formulas (7.2) (i), (ii) hold, but $2 f_{k}(X)<k n$ and $n \neq(k+1) f$.

For instance, take $X$ to be the Segre variety $\operatorname{Seg}(3,4)$ in $\mathbb{P}^{19}$. The variety $S^{2}(X)$ has dimension 17. The first tangential projection sends $X$ to $\operatorname{Seg}(2,3) \subset \mathbb{P}^{11}$. The second tangential projection sends $X$ to $\operatorname{Seg}(1,2) \subset \mathbb{P}^{5}$. One computes $f=f_{1}=\gamma_{1}=\psi_{1}=2$, $\gamma_{2}=\psi_{2}=4=2 f, f_{2}=6=3 f$. Moreover, using Proposition 5.13 one sees that $X$ is an $R_{2}$-variety.

On the other hand, $n=7 \neq 6=(k+1) f$, and $2 f_{k}=12<14=n k$.

We can now prove our extension of Zak's linear normality theorem.

Theorem 7.5. Let $X \subset \mathbb{P}^{r}$ be a non-degenerate variety of dimension n. Assume $X$ is an $R_{k}$-variety (or $X$ is $k$-smooth). Then

$$
\text { either } \quad S^{k}(X)=\mathbb{P}^{r} \quad \text { or } \quad s^{(k)}(X) \geq \frac{k+2}{2} n+k
$$


Proof. Let $s^{(k)}(X)<r$. By [7.1), one has

$$
s^{(k)}(X)=(k+1) n+k-f_{k} \geq(k+1) n+k-\frac{k n}{2}=\frac{k+2}{2} n+k .
$$

Corollary 7.6. Let $X \subset \mathbb{P}^{r}$ be a $k$-smooth variety of dimension $n$. If $(k+2) n>2(r-k)$ then $X$ is linearly normal.

Proof. If $X$ is not linearly normal, then it comes as an isomorphic projection of a variety $X^{\prime} \subset \mathbb{P}^{r+1}$ from a point $p \notin X^{\prime}$. Hence $X^{\prime}$ is also $k$-smooth and therefore an $R_{k}$-variety. Then Theorem 7.5 implies that $S^{k}\left(X^{\prime}\right)=\mathbb{P}^{r+1}$. Therefore there is some $(k+1)$-secant $k$-space to $X^{\prime}$ passing through the centre of projection, yielding a $(k+1)$-secant $(k-1)$ space to $X$, contradicting $k$-smoothness.

We finish this section by stressing that the $R_{k}$-property in Theorem 7.5 is really essential, as the following example due to C. Fontanari shows.

Example 7.7. Consider the rational normal scroll 3 -fold $X:=S(1,1, h) \subset \mathbb{P}^{h+4}, h \geq 2$. Note that we have two line sections on $X$ spanning a 3 -space $\Pi$. For all $k \geq 1, S^{k}(X)$ is the cone with vertex $\Pi$ over $S^{k}(Y)$, with $Y$ a rational normal curve in an $h$-space $\Pi^{\prime}$ which is skew with $\Pi$. Therefore

$$
s^{(k)}(X)=2 k+5<h+4
$$

as soon as $k<(h-1) / 2$. On the other hand $2 k+5$ is smaller than the bound $\frac{k+2}{2} n+k=$ $\frac{5}{2} k+3$ as soon as $k>4$.

Indeed, $X$ is not an $R_{k}$-variety. For instance, consider the case $h=2 k+2$. Then there is a unique hyperplane $H$ which is tangent to $X$ at $k+1$ general points $p_{0}, \ldots, p_{k}$ : it contains $\Pi$ and projects to the unique hyperplane $H^{\prime}$ in $\Pi^{\prime}$ which is tangent to $Y$ at the projection points $p_{0}^{\prime}, \ldots, p_{k}^{\prime}$ of $p_{0}, \ldots, p_{k}$ from $\Pi$. The $k$-contact locus $\Sigma$ contains the union of the rulings of the scroll $X$ passing through $p_{0}, \ldots, p_{k}$. Suppose $H$ is $J_{k}$-tangent to $X$ along $\Sigma$. By projecting down from three general points of $\Pi$ we would have $J_{k-3^{-}}$ tangency of the image hyperplane to a cone over $Y$, a contradiction (see Lemma 5.11 and Example 4.3 .

\section{The classification theorem}

Let us start with the following definition.

Definition 8.1. A $k$-Severi variety is an irreducible, non-degenerate $R_{k}$-variety $X \subset \mathbb{P}^{r}$ such that

$$
r>s^{(k)}(X)=\frac{k+2}{2} n+k .
$$

A 1-Severi variety is simply called a Severi variety. Note we do not require smoothness of $X$ in Definition 8.1 We will see in a moment that $k$-Severi varieties are smooth, thus, in case $k=1$, our definition of Severi varieties turns out to coincide with the one of Zak (see [27]). 
Remark 8.2. A trivial, but useful, remark is that $k$-Severi varieties are not cones, because of the $R_{k}$-property.

Another striking result of Zak's is the famous classification theorem.

Theorem 8.3 (Zak's Classification Theorem). Let $X \subseteq \mathbb{P}^{r}$ be a smooth Severi variety. Then $X$ is one of the following varieties:

(i) the Veronese surface $V_{2,2}$ in $\mathbb{P}^{5}$;

(ii) the 4-dimensional Segre variety $\operatorname{Seg}(2,2)$ in $\mathbb{P}^{8}$;

(iii) the 8-dimensional Grassmann variety $\mathbb{G}(1,5)$ in $\mathbb{P}^{14}$;

(iv) the 16-dimensional E-variety in $\mathbb{P}^{26}$.

Remark 8.4. Case (i) of Theorem 8.3 is due to Severi (see [24]), whence the name of Severi varieties.

Recall that Severi varieties are related to the unitary composition algebras $\mathbb{R}, \mathbb{C}, \mathbb{H}, \mathbb{O}$. If $\mathcal{A}$ is one of these algebras, then take all $3 \times 3$ hermitian matrices $A$ and impose that $\operatorname{rk}(A)=1$. This gives equations defining the Severi varieties. The secant variety to a Severi variety is defined by the vanishing of $\operatorname{det}(A)$. Note that $\mathbb{O}$ being non-associative, the existence of this determinant is somewhat exceptional. Indeed there is no analogue for higher order matrices.

We devote this section to the analogous classification of $k$-Severi varieties for $k \geq 2$.

Lemma 8.5. Let $X \subset \mathbb{P}^{r}$ be a $k$-Severi variety. Let $p_{0}, \ldots, p_{k} \in X$ be general points and $x \in\left\langle p_{0}, \ldots, p_{k}\right\rangle$ be a general point of $S^{k}(X)$. Then $\Psi_{p_{0}, \ldots, p_{k}}$ and $E_{k, x}$ are irreducible components of $\Gamma_{p_{0}, \ldots, p_{k}}$.

Proof. This follows from (7.2)(i) of Lemma 7.1

Lemma 8.6. Let $X \subset \mathbb{P}^{r}$ be a $k$-Severi variety. Let $p_{0}, \ldots, p_{k} \in X$ be general points and set, as usual, $\Gamma_{i}=\Gamma_{p_{0}, \ldots, p_{i}}$ and $\Pi_{i}=\left\langle\Gamma_{i}\right\rangle$. Then for all $i=1, \ldots, k$ one has

$$
T_{\Gamma_{i}, p_{1}, \ldots, p_{i}}=T_{X, p_{1}, \ldots, p_{i}} \cap \Pi_{i}
$$

Moreover the intersection of $\Pi_{i}$ with $X$ coincides with $\Gamma_{i}$.

Proof. One has

$$
T_{\Gamma_{i}, p_{1}, \ldots, p_{i}} \subseteq T_{X, p_{1}, \ldots, p_{i}} \cap \Pi_{i}
$$

By formulas (7.2) and Proposition 3.9, $T_{\Gamma_{i}, p_{1}, \ldots, p_{i}}$ has codimension 1 in $\Pi_{i}$. Hence, if 8.2 did not hold, then $T_{X, p_{1}, \ldots, p_{i}}$ would contain $\Pi_{i}$, and therefore $\Gamma_{i}$. Thus it would contain $p_{0}$, i.e. a general point of $X$, a contradiction. The final assertion follows from the fact that $\Gamma_{i}$ is the general fibre of the general tangential projection $\tau_{i-1}$.

Theorem 8.7. Let $X \subset \mathbb{P}^{r}$ be a $k$-Severi variety of dimension $n$. Then $X$ is smooth. 
Proof. After a general projection, we may assume that $r=\frac{k+2}{2} n+k+1$.

Let $p_{1}, \ldots, p_{k} \in X$ be general points. Let $\tau_{k}$ be the $k$-tangential projection from $T_{X, p_{1}, \ldots, p_{k}}$. Its image $X_{k}$ has dimension $n-\psi_{k}$ and spans a projective space of dimension $r-1-s^{(k-1)}=r-1-\left(k n+k-1-f_{k-1}\right)$. Using (7.2) one sees that $X_{k}$ is a non-linear hypersurface in $\mathbb{P}^{f+1}$.

Let $q_{0}, q_{1} \in X$ be general points. By 77.2 , and since the general secant line to $X$ is not a trisecant (see [27]), the tangential 1-contact locus $\Gamma_{1}=\Gamma_{q_{0}, q_{1}}$ is an $f$-dimensional quadric. Indeed, by Proposition 3.9 we have $\operatorname{dim}\left(\Pi_{1}\right)=f+1$.

Claim 8.8. The tangential projection $\tau_{k}$ isomorphically maps $\Gamma_{1}$ to $X_{k}$. Then $X_{k}$ and $\Gamma_{1}$ are smooth quadrics. Moreover the general tangential 1-contact locus intersects the general tangential $k$-contact locus $\Gamma_{k}=\Gamma_{p_{0}, \ldots, p_{k}}$ transversally in one point.

Proof of the Claim. In order to prove the first assertion, it suffices to show that $\Pi_{1}=\left\langle\Gamma_{1}\right\rangle$ does not intersect $T_{X, p_{1}, \ldots, p_{k}}$. Aiming for a contradiction, assume that $T_{X, p_{1}, \ldots, p_{k}} \cap \Pi_{1}$ $\neq \emptyset$. If this happens, then either

(i) $\tau_{k}\left(\Gamma_{1}\right)$ is a subspace of dimension at most $f$, or

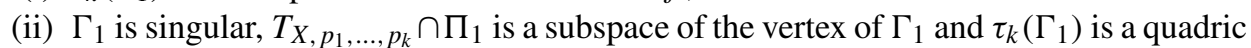
of dimension smaller than $f$.

Case (i) is impossible. Indeed, given two general points of $X_{k}$ there would be a subspace $\tau_{k}\left(\Gamma_{1}\right)$ containing them and sitting inside $X_{k}$, contradicting the non-degeneracy of $X_{k}$ in $\mathbb{P}^{f+1}$. In case (ii), the general tangential $k$-contact locus $\Gamma_{k}$, i.e. the general fibre of $\tau_{k}$, intersects the singular quadric $\Gamma_{1}$ in a positive-dimensional subspace strictly con-

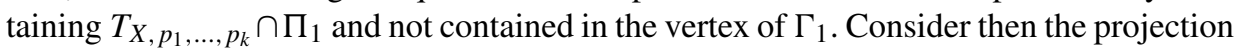
$\pi: X \rightarrow \mathbb{P}^{n}$ from $\Pi_{k}=\left\langle\Gamma_{k}\right\rangle$ and let $X^{\prime}$ be its image, which is non-degenerate in $\mathbb{P}^{n}$.

By the above considerations, the image of $\Gamma_{1}$ via $\pi$ would be a linear subspace of dimension $f^{\prime}<f$. Therefore $\operatorname{dim}\left(X^{\prime}\right)<n$ and moreover two general points of $X^{\prime}$ would be contained in a linear subspace of dimension $f^{\prime}$ contained in $X^{\prime}$. This contradicts the non-degeneracy of $X^{\prime}$.

As for the second assertion, note that $X_{k}$ is a quadric, which is smooth, otherwise we have a contradiction to Lemma 8.5 The final assertion also follows from Lemma 8.5 since the above argument implies that $\Gamma_{1}$ intersects the general fibre of $\tau_{k}$, i.e. $\Gamma_{k}$, transversally in one point.

As a consequence we have:

Claim 8.9. For all $i=2, \ldots, k$, the general tangential $i$-contact locus $\Gamma_{i}$ is an $(i-1)$ Severi variety.

Proof of the Claim. The irreducibility of the general 1-contact loci implies the irreducibility of the higher contact loci $\Gamma_{i}$ with $i \geq 2$. Moreover, by Lemma 8.6 and by the $R_{k}$ property, $\Gamma_{i}$ is an $R_{i-1}$-variety, and Lemma 7.3 and Proposition 3.9 imply that $\Gamma_{i}$ is a $(i-1)$-Severi variety.

Next we have: 
Claim 8.10. For all $i=2, \ldots, k$, and for the general tangential $i$-contact locus $\Gamma_{i}$, the secant variety $S^{i-1}\left(\Gamma_{i}\right)$ is not a cone. Similarly $S^{k}(X)$ is not a cone.

Proof of the Claim. We prove the assertion for $\Gamma_{i}$, the proof for $X$ is similar. Suppose $S^{i-1}\left(\Gamma_{i}\right)$ is a cone with vertex $p$. Since $\Gamma_{i}$ is not a cone (see Remark 8.2), there is a maximum positive integer $j<i$ such that $p$ is not a vertex of $S^{j-1}\left(\Gamma_{i}\right)$. Then $p$ does not sit in the indeterminacy locus of the general projection $\tau_{j}=\tau_{X, p_{1}, \ldots, p_{j}}$. The image $Z$ of $\Gamma_{i}$ via $\tau_{j}$ has dimension $(i-j) f$ and is a cone with vertex at the image of $p$. Hence the general tangent hyperplane to $Z$ is tangent along a positive-dimensional variety. This implies that the general tangential $j$-contact locus of $\Gamma_{i}$, hence of $X$, has dimension at least $j f+1$, which is a contradiction.

Remark 8.11. The previous argument provides a sort of converse to the criterion of Proposition 5.13

Indeed, it proves that if an $R_{k}$-variety satisfies $\gamma_{i}=\psi_{i}=i f$ for all $i=1, \ldots, k$, then the intersection of the indeterminacy loci of all tangential projections $\tau_{p_{0}, \ldots, p_{i}}$ is empty for $i=1, \ldots, k$.

Now we improve Lemma 8.6

Claim 8.12. For all $i=2, \ldots, k$, the general tangential $i$-contact locus $\Gamma_{i}$ is smooth. Furthermore $X$ is smooth along $\Gamma_{k}$, and $\Gamma_{k}$ is the schematic intersection of $\Pi$ with $X$.

Proof of the Claim. Suppose $\Gamma_{i}$ is singular at a point $p$. Consider the general tangential projection $\tau_{i}=\tau_{X, p_{1}, \ldots, p_{i}}$. By generic smoothness of $\tau_{i}$ and by Lemma $8.5, p$ has to be in the indeterminacy locus of $\tau_{i}$, i.e. in the intersection of $T_{X, p_{1}, \ldots, p_{i}}$ with $\Gamma_{i}$. By Lemma 8.6 this coincides with the intersection of $T_{\Gamma_{i}, p_{1}, \ldots, p_{i}}$ with $\Gamma_{i}$. By the genericity of $p_{1}, \ldots, p_{i}$ and Terracini's Lemma, we deduce that $S^{i-1}\left(\Gamma_{i}\right)$ is a cone with vertex $p$, contradicting Claim 8.10

Let now $q \in \Gamma_{k}$ be any point and let $p_{1}, \ldots, p_{k} \in \Gamma_{k}$ be general points. Thus $p_{1}, \ldots, p_{k}$ are general points on $X$. Moreover $\Gamma_{k}$ is the fibre of $q$ in the tangential projection $\tau_{k}$. Since the image $X_{k}$ of $X$ via $\tau_{k}$ is smooth of dimension $f$ and the fibre of $q$ via $\tau_{k}$ is smooth of dimension $\psi_{k}=k f$ at $q$ we see that $X$ is smooth of dimension $n=(k+1) f$ at $q$. The final assertion follows by the same argument.

Now we go back to the projection $\pi: X \rightarrow \mathbb{P}^{n}$ from $\Pi_{k}$.

Claim 8.13. The map $\pi: X \rightarrow \mathbb{P}^{n}$ is birational.

Proof of the Claim. Let $X^{\prime}$ be the image of $X$. By Claim 8.8 , the restriction of $\pi$ to a general tangential 1-contact locus $\Gamma_{1}$ is birational, and its image is an $f$-subspace of $\mathbb{P}^{n}$ contained in $X^{\prime}$. This shows that $X^{\prime}$ is a subspace of $\mathbb{P}^{n}$, and therefore $X^{\prime}=\mathbb{P}^{n}$.

Assume towards a contradiction that $\pi$ is not birational. Then, if $x \in X$ is a general point, there is a point $y \in X$, with $x \neq y$, such that $\pi(x)=\pi(y)$. Note that there is some $f$-dimensional quadric $\Gamma$ containing $x$ and $y$ and contained in $X$, i.e. a flat limit of $\Gamma_{x, z}$ with $z$ a general point of $X$ tending to $y$. The quadric $\Gamma$, as well as $\Gamma_{x, z}$, has a non-empty intersection with $\Pi_{k}$ (see Claim 8.8). Since the fibre of $x$ in $\pi$ is 0 -dimensional, also the 
fibre of $\pi_{\mid \Gamma}$ is finite. This implies that $\langle\Gamma\rangle \cap \Pi_{k}$ is only one point $z \in \Gamma$ and that the line $\langle x, z\rangle$ is not contained in $\Gamma$. So the line $\langle x, z\rangle$ meets $\Gamma$ only at $z$ and $x$, contradicting $\pi(x)=\pi(y)$.

Let $H$ be the hyperplane in $\mathbb{P}^{\frac{k+2}{2} n+k+1}$ which is tangent to $X$ along $\Gamma_{k}$ and let $H^{\prime}$ be its image via $\pi$.

Claim 8.14. The inverse of the map $\pi: X \rightarrow \mathbb{P}^{n}$ is well defined off $H^{\prime}$.

Proof of the Claim. We resolve the indeterminacies of $\pi$ by blowing-up $\Gamma_{k}$. If $f$ : $\tilde{X} \rightarrow X$ is this blow-up, then $p=\pi \circ f: \tilde{X} \rightarrow \mathbb{P}^{n}$ is a morphism. Note that $\tilde{X}$ is smooth along the exceptional divisor $E$ by Claim 8.12 Points on the exceptional divisor $E$ are mapped via $p$ to points of $H^{\prime}$.

Let $z \in \mathbb{P}^{n}$ be a point where the inverse of $\pi$ is not defined. If $x \in X$ is a point such that $\pi(x)=z$, then the subspace $\Pi_{x}:=\left\langle\Pi_{k}, x\right\rangle$ intersects $X$ along an irreducible positive-dimensional subvariety $Z$ containing $x$, which is contracted to a point via $\pi$. Since $\Pi_{k} \cap X=\Gamma_{k}$ (see Corollary 8.6), we have $Z \cap \Gamma_{k} \neq \emptyset$. Let $Z^{\prime}$ be the strict transform of $Z$ on $\tilde{X}$. Then $Z^{\prime}$ intersects $E$, and is contracted to a point by $p$. Hence $z \in H^{\prime}$.

Now we are able to finish the proof of Theorem 8.7. By Claim 8.14, $X-(H \cap X)$ is isomorphic to the affine space $\mathbb{P}^{n}-H^{\prime}$. It remains to prove that there is no point $x \in X$ contained in all hyperplanes tangent to the tangential $k$-contact loci $\Gamma_{k}$. Suppose that such a point exists. Let $p_{1}, \ldots, p_{k} \in X$ be general points and consider again the tangential projection $\tau_{k}$ from $T_{X, p_{1}, \ldots, p_{k}}$. Since $S^{k-1}(X)$ is not a cone (see Claim 8.10, $\tau_{k}$ is well defined at $p$. Let $z$ be its image via $\tau_{k}$. Then all tangent hyperplanes to $X_{k}$ would contain $z$, hence $X_{k}$ would be a cone, a contradiction.

We can now prove the classification theorem. By taking into account Theorem 8.7 and Zak's Classification Theorem 8.3 , we may consider only $k$-Severi varieties with $k \geq 2$.

Theorem 8.15. Let $X \subseteq \mathbb{P}^{r}$ be a $k$-Severi variety with $k \geq 2$. Then $X$ is one of the following varieties:

(i) the $(k+1)$-dimensional Veronese variety $V_{2, k+1}$ in $\mathbb{P}^{k(k+3) / 2}$;

(ii) the $2(k+1)$-dimensional Segre variety $\operatorname{Seg}(k+1, k+1)$ in $\mathbb{P}^{k^{2}+4 k+3}$;

(iii) the $4(k+1)$-dimensional Grassmann variety $\mathbb{G}(1,2 k+3)$ in $\left.\mathbb{P}^{(2 k+4} \begin{array}{c}2 \\ 2\end{array}\right)-1$.

Proof. The varieties in (i)-(iii) are $R_{k}$-varieties (see Example 5.14). Moreover they are $k$-Severi varieties, i.e. (8.1) holds for them (see [27]).

Set now $s=s^{(k)}+1=\frac{k+2}{2} n+k+1$ and take a general projection $X^{\prime}$ to $\mathbb{P}^{s}$. Then $X^{\prime}$ is still a $k$-Severi variety. Moreover we have $k_{0}\left(X^{\prime}\right)=k+1, \psi_{i}\left(X^{\prime}\right)=\psi_{i}(X)=i f$, $f_{i}\left(X^{\prime}\right)=f_{i}(X)=\frac{i(i+1)}{2} f$ for all $i=1, \ldots, k$ and $n=(k+1) f$ by Lemma 7.3. Thus $X^{\prime}$ is a Scorza variety. The classification follows from Zak's classification of Scorza varieties, which implies that Scorza varieties are linearly normal, in particular $X=X^{\prime}$. Notice the smoothness of the entry loci, which follows by Lemma 8.5 and Claims 8.9 and 8.12 . This is essential in Zak's argument (see Remark 3.17). 
Remark 8.16. The $k$-Severi varieties, with $k \geq 2$, are related to the unitary composition algebras $\mathbb{R}, \mathbb{C}, \mathbb{H}$. In this case we take all $(k+1) \times(k+1)$ hermitian matrices $A$ and impose that $\operatorname{rk}(A)=1$. This gives the equations defining the $k$-Severi varieties. Again the $k$-secant variety to a $k$-Severi variety is defined by the vanishing of $\operatorname{det}(A)$. The absence of the analogue of the $E_{6}$-variety, related to the composition algebra $\mathbb{O}$, reflects the absence of higher order determinants on $\mathbb{O}$ (see Remark 8.4).

A quick proof of the classification of Severi varieties can be obtained by using the beautiful ideas contained in [21]. Along the same lines one can give a proof of the classification of $k$-Severi varieties, alternative to the one described above based on Zak's classification of Scorza varieties. We do not dwell on this here.

\section{Speculations}

Perhaps the main motivation for Zak's beautiful piece of work was the following famous conjecture by Hartshorne (see [14]):

Conjecture 9.1 (Hartshorne's conjecture). Let $X \subset \mathbb{P}^{r}$ be a smooth, irreducible, nondegenerate variety of dimension $n$. If $3 n>2 r$ then $X$ is a complete intersection of $r-n$ hypersurfaces in $\mathbb{P}^{r}$.

This in turn was motivated by Barth-Larsen's fundamental result (see [2]) to the effect that smooth varieties $X \subset \mathbb{P}^{r}$ of low codimension are topologically similar to $\mathbb{P}^{r}$. BarthLarsen's theorem, in our context, can be stated as follows:

Theorem 9.2. Let $X \subset \mathbb{P}^{r}$ be a smooth, irreducible variety. Then for any non-negative integer $i<f_{1}(X)$ the natural map

$$
\rho_{X, i}: H^{i}\left(\mathbb{P}^{r}, \mathbb{Z}\right) \rightarrow H^{i}(X, \mathbb{Z})
$$

is an isomorphism. In particular, if $f_{1}(X) \geq 2$ then $X$ is simply connected and if $f_{1}(X) \geq 3$ then $\operatorname{Pic}(X)$ is generated by the hyperplane class.

One of the basic steps in the proof of Conjecture 9.1 would be to show that, under the hypotheses, $X$ is projectively Cohen-Macaulay. Since linear normality is the first naïve requirement for being projectively Cohen-Macaulay, this is the motivation for Conjecture 7.2, which in turn motivates Zak's theorems.

Now, in presence of our refined form of Zak's linear normality theorem, one may speculate on the possibility of having an even more general view on Hartshorne's conjecture. This is what we want to present next. To be precise, we want to propose the following:

Conjecture 9.3 (Extended Hartshorne's conjecture). There is a suitable function $f(r, n, k)$ such that the following happens. Let $X \subset \mathbb{P}^{r}$ be a $k$-smooth, irreducible, nondegenerate variety of dimension $n$. If $(k+2) n>2 r$ then $\mathscr{I}_{X}$ is generated by at most $f(r, n, k)$ elements.

This conjecture does not make too much sense unless one specifies the form of the function $f(r, n, k)$. What we intend is that $f(r, n, k)$ should be reasonably small. If one wants to be really bold, one may even conjecture that $f(r, n, k)=k(r-n)$. A further strength- 
ening of the conjecture would be to replace the ideal sheaf $\mathscr{I}_{X}$ with the homogeneous ideal $I_{X}$.

Example 9.4. There are varieties at the boundary of Hartshorne's conjecture. One of them is $\mathbb{G}(1,4)$, which has dimension 6 in $\mathbb{P}^{9}$. Its homogeneous ideal is generated by five quadrics. This would fit Conjecture 9.3 for $k=2$ and $f(r, n, 2)=2(r-n)$, but unfortunately $\mathbb{G}(1,4)$ is not 2 -smooth, since it contains lines.

Another variety at the boundary of Hartshorne's conjecture is the 10-dimensional spinor variety $S_{4} \subset \mathbb{P}^{15}$, its homogeneous ideal being generated by 10 quadrics. Again this would fit Conjecture 9.3 for $k=2$ and $f(r, n, 2)=2(r-n)$, but this variety is not 2-smooth either.

More varieties at the boundary of Hartshorne's conjecture, actually all the known ones, are deduced from these by pulling them back via a general morphism $\mathbb{P}^{r} \rightarrow \mathbb{P}^{r}$. Now these can in general be 2-smooth and Conjecture 9.3 holds for them with $f(r, n, 2)$ $=2(r-n)$.

The examples of $\mathbb{G}(1,4)$ and $S_{4}$ suggest that the $k$-smoothness assumption in Conjecture 9.3 might even be too strong. Maybe something like the $R_{k}$-property could suffice.

Remark 9.5. At this point a related natural question arises: is there, in this same spirit, any extension of Barth-Larsen's Theorem 9.2? By taking into account 3.1) we see that

$$
2 f_{k} \geq k(k+1) f_{1}
$$

and therefore one might ask: is the map $\rho_{X, i}$ an isomorphism for all positive integers $i$ such that $k(k+1)(i+1) \leq 2 f_{k}(X)$, under the assumption that $X \subset \mathbb{P}^{r}$ be a $k$-smooth, irreducible variety? Or, is $\rho_{X, i}$ an isomorphism under the condition

$$
\left(\begin{array}{c}
k+1 \\
2
\end{array}\right) i \leq(k+1) n-r-\left(\begin{array}{l}
k \\
2
\end{array}\right)
$$

if $X \subset \mathbb{P}^{r}$ is a $k$-smooth, irreducible variety?

\section{References}

[1] Adlansdvik, B.: Joins and higher secant varieties. Math. Scand. 61, 213-222 (1987) Zbl 0657.14034 MR 0947474

[2] Barth, W., Larsen, M. F.: On the homotopy groups of complex projective algebraic manifolds. Math. Scand. 30, 88-94 (1972) Zbl 0238.32008 MR 0340643

[3] Beltrametti, M., Sommese, A.: Zero-cycles and $K$-th order embeddings of smooth projective surfaces. Symposia Math. 32, 33-48 (1991) Zbl 0827.14029||MR 1273371

[4] Catalisano, M. V., Geramita, A. V., Gimigliano, A.: Ranks of tensors, secant varieties of Segre varieties and fat points. Linear Algebra Appl. 355, 263-285 (2002) Zbl 1059.14061 MR 1930149

[5] Chiantini, L., Ciliberto, C.: Weakly defective varieties. Trans. Amer. Math. Soc. 354, 151-178 (2002) Zbl 1045.14022 MR 1859030

[6] Chiantini, L., Ciliberto, C.: Threefolds with degenerate secant variety: on a theorem of G. Scorza. In: Geometric and Combinatorial Aspects of Commutative Algebra (Messina, 1999), Lecture Notes in Pure Appl. Math. 217, Dekker, 111-124 (2001) Zbl 1048.14034 MR 1824221 
[7] Chiantini, L., Ciliberto, C.: On the classification of defective threefolds. In: Projective Varieties with Unexpected Properties (Siena, 2004), C. Ciliberto et al. (eds.), de Gruyter, 131-176 (2005) Zbl 1100.14044 MR 2202251

[8] Chiantini, L., Ciliberto, C.: On the concept of $k$-secant order of a variety. J. London Math. Soc. (2) 73, 436-454 (2006) Zbl 1101.14067 MR 2225496

[9] Ciliberto, C.: Geometric aspects of polynomial interpolation in more variables and of Waring's problem. In: European Congress of Mathematics (Barcelona, 2000), Vol. 1, Progr. Math. 201, Birkhäuser, 289-316 (2001) Zbl 1078.14534 MR 1905326

[10] Dale, M.: Terracini's lemma and the secant variety of a curve. Proc. London Math. Soc. 49, 329-339 (1984) Zbl 0571.14025 MR 0748993

[11] Fujita, T., Roberts, J.: Varieties with small secant varieties: the extremal case. Amer. J. Math. 103, 953-976 (1981) Zbl 0475.14046 MR 0630774

[12] Fulton, W., Hansen, J.: A connectedness theorem for projective varieties, with applications to intersections and singularities of mappings. Ann. of Math. (2) 110, 159-166 (1979) Zbl 0389.14002 MR 0541334

[13] Garcia, L. D., Stillman, M., Sturmfels, B.: Algebraic geometry of bayesian networks. J. Symbolic Comput. 39, 331-355 (2005) Zbl 1126.68102 MR 2168286

[14] Hartshorne, R.: Varieties with small codimension in projective space. Bull. Amer. Math. Soc. 80, 1017-1032 (1974) Zbl 0304.14005 MR 0384816

[15] Iarrobino, A., Kanev, V.: Power Sums, Gorenstein Algebras, and Determinantal Loci. Lecture Notes in Math. 1721, Springer (1999). Zbl 0942.14026 MR 1735271

[16] Ionescu, P., Russo, F.: Conic-connected manifolds. J. Reine Angew. Math., to appear; mathAG/0701885

[17] Muñoz, R., Sierra, J. C., Solá Conde, L. E.: Tangential projections and secant defective varieties. Bull. London Math. Soc. 39, 949-961 (2007) Zbl 1143.14041 MR 2392818

[18] Palatini, F.: Sulle superficie algebriche i cui $S_{h}(h+1)$-seganti non riempiono lo spazio ambiente. Atti Accad. Torino 41, 634-640 (1906) Zbl 37.0667.01

[19] Palatini, F.: Sulle varietà algebriche per le quali sono di dimensione minore dell'ordinario, senza riempire lo spazio ambiente, una o alcune delle varietà formate da spazi seganti. Atti Accad. Torino 44, 362-374 (1909) JFM 40.0713.01

[20] Ranestad, K., Schreier, F.: Varieties of sums of powers. J. Reine Angew. Math. 525, 147-181 (2000) Zbl 1078.14506 MR 1780430

[21] Russo, F.: Varieties with quadratic entry locus. I. Math. Ann. 344, 597-617 (2009) Zbl 1170.14040 MR 2501303

[22] Scorza, G.: Determinazione delle varietà a tre dimensioni di $S_{r}, r \geq 7$, i cui $S_{3}$ tangenti si intersecano a due a due. Rend. Circ. Mat. Palermo 25, 193-204 (1908) JFM 39.0717.01

[23] Scorza, G.: Sulle varietà a quattro dimensioni di $S_{r}(r \geq 9)$ i cui $S_{4}$ tangenti si tagliano a due a due. Rend. Circ. Mat. Palermo 27, 148-178 (1909) JFM 40.0712.01

[24] Severi, F.: Intorno ai punti doppi impropri di una superficie generale dello spazio a quattro dimensioni e ai suoi punti tripli apparenti. Rend. Circ. Mat. Palermo 15, 33-51 (1901) JFM 32.0648.04

[25] Terracini, A.: Sulle $V_{k}$ per cui la varietà degli $S_{h},(h+1)$-seganti ha dimensione minore dell'ordinario. Rend. Circ. Mat. Palermo 31, 392-396 (1911) JFM 42.0673.02

[26] Zak, F. L.: Projections of algebraic varieties. Math. USSR-Sb. 44, 535-544 (1983) Zbl 0511.14026 MR 0665860

[27] Zak, F. L.: Tangents and Secants of Varieties. Transl. Math. Monogr. 127, Amer. Math. Soc. (1993) Zbl 0795.14018 MR 1234494 